rev.relac.int.estrateg.segur.8(1):45-60,2013

\title{
EL MULTILATERALISMO ACTUAL: CRISIS Y DESAFÍOS*
}

\section{Camilo Zambrano Jáuregui ${ }^{* *}$}

\section{RESUMEN}

El multilateralismo ha estado cargado por algunas contradicciones de origen; se describe como un concepto no geográfico, no obstante su aplicación ha estado plasmada por temas de geopolítica y geo-estrategia. El sistema internacional también enfrenta cambios significativos y que tienen consecuencias de carácter estructural. Algunos de estos cambios incluyen la reconfiguración de las fuerzas militares y sus misiones vitales, la recomposición de la economía mundial y el respectivo peso que las diferentes naciones tienen y la creciente evidencia de un papel limitado de Naciones Unidas y sus organizaciones. En este sentido, cabe preguntarse entonces si la nueva

* El presente artículo es parte de la investigación del Centro de Estudios Estratégicos de la ESPE, línea de investigación L.I. 13: Seguridad y Defensa, sub-línea: Relaciones internacionales.

** Docente e investigador en relaciones internacionales y análisis de conflictos del Centro de Estudios Estratégicos de la ESPE.Docente de la Universidad San Francisco de Quito. Se desempeñó como asesor en relaciones internacionales en la Secretaría Nacional de Inteligencia y como asistente de investigación en el Woodrow Wilson Center y el Instituto de Paz en Washington, D.C. Magister en Relaciones Internacionales y Resolución de Conflictos de la American University - Washington, D.C. y Licenciaturas en Relaciones Internacionales y Finanzas en la USFQ. camilozambranoj@gmail.com 
configuración del sistema y los desafíos que estos suponen también exige llevar a cabo modificaciones y reformas profundas al multilateralismo, tal como lo conocemos hoy en día, concluyendo que las potencias emergentes deben asumir su rol de liderazgo y afrontar los costos implícitos con el nuevo rol que les compete.

Palabras Clave: Multilateralismo, crisis, potencias emergentes, geo-política, gobernanza

\title{
PRESENT-DAY MULTILATERALISM: CRISIS AND CHALLENGES
}

\begin{abstract}
Multilateralism has been loaded with some contradictions of origin; it is described as a nongeographic concept, though its implementation has been shaped by issues of geopolitics and geostrategy. The international system also faces significant changes that have structural consequences. Some of these changes include the reconfiguration of the military and their vital missions, the restructuring of the global economy and the respective weight that different nations have and the growing evidence of a limited role of the $\mathrm{UN}$ and its organizations. In this regard, the question is then whether the new configuration of the system and the resulting challenges also require deep changes to multilateralism, as we know it today, concluding that emerging powers must assume a leadership role and disburse the costs implicit with the new role they are taking.
\end{abstract}

Key words: Multilateralism, crisis, emerging power, geo-politics, governance

\section{MULTILATERALISMO ATUAL: CRISE E DESAFIOS}

\section{RESUMO}

O multilateralismo sempre teve algumas contradições de origem. Ele é descrito como um conceito não geográfico, porém a sua aplicação tem sido moldada por questões de geopolítica e geoestratégia. O sistema internacional também se depara com mudanças significativas, que têm consequências estruturais. Algumas dessas mudanças incluem a reconfiguração dos militares e de suas missões vitais, a reestruturação da economia global e o respectivo peso que as diferentes nações têm, e a crescente evidência de um papel limitado da ONU e de suas organizações. Neste sentido, a questão é se a nova configuração do sistema e os desafios que isso implica, também demanda realizar modificações e reformas profundas no multilateralismo, como o conhecemos hoje em dia, e se conclui que as potências emergentes devem assumir um papel de liderança e enfrentar os custos decorrentes do novo papel que lhes pertence.

Palavras-chave:multilateralismo, crise,potências emergentes, geopolítica, governança 


\section{INTRODUCCIÓN}

El multilateralismo y las normas internacionales han demostrado tener gran capacidad para cambiar o al menos influir en los procesos de transición del sistema internacional (Ruggie, 1992). Apenas 10 años antes de la caída del muro de Berlín y el colapso de la Unión Soviética,analistas internacionales y académicos especializados predecían que la transición pacífica era poco probable (Gilpin, 1981:15).

Desde su conceptualización inicial, el multilateralismo ha estado cargado por algunas contradicciones de origen; se describe como un concepto no geográfico o, mejor dicho,incluyente - casi universal, no obstanteque su aplicación haya estado plasmado por temas de geopolítica y geoestrategia, particularmente debido a que desde sus inicios con el Presidente Teodore Roosevelt el multilateralismo ha consistido en una forma de validar o legitimar internacionalmente las políticas de ciertos países.

El multilateralismo, de una manera sencilla,es una forma de toma de decisiones en donde el consenso y la negociación con varias partes son esenciales. Según Robert Keohane (1990:731), "el multilateralismo es la práctica para coordinar políticas nacionales en grupos de tres o más estados" y John Ruggie (1992:566) complementa esta definición indicando que lo que hace distintivo al multilateralismo "no es sólo su capacidad para coordinar políticas nacionales entre tres o más países, que es algo que otras formas organizacionales hacen, sino que lo hace con base en ciertos principios de relacionamiento entre los estados".

No cabe duda que Estados Unidos, como superpotencia económica y militar, fue parte de la construcción y diseño de las instituciones que ahora conocemos y utilizamos como foros multilaterales. Luego de la desaparición de la Unión Soviética, la influencia estadounidense incluso tomó más fuerza. Como hegemón, el impacto que ha tenido Estados Unidos es limitar la posibilidad de que las instituciones y regímenes multilaterales se conviertan en foros que fomenten elmultipolarismo. De hecho, es innegable que inclusive en el contexto de la crisis financiera actual su influencia para proteger sus propios intereses es todavía definitiva.

Sin embargo, el sistema internacional también enfrenta cambios significativos y que tienen consecuencias de carácter estructural.Algunos de estos cambios incluyen la reconfiguración de las fuerzas militares y sus misiones vitales, la recomposición de la economía mundial y el respectivo peso que las diferentes naciones tienen y la creciente evidencia de un papel limitado de Naciones Unidas y sus organizaciones. Como reconocen ciertos analistas, los cambios drásticos del sistema internacional han favorecido la intervención militar con o sin el consentimiento del Consejo de Seguridad de Naciones Unidas (Kardas, 2001).

Bajo este escenario, la cuestión que se plantea ahora es examinar qué implicaciones tiene para la cooperación internacional los cambios drásticos que se están viviendo en el sistema 
internacional e identificar cómo desarrollar mecanismos adecuados para hacer frente a dichos cambios y a los nuevos desafíos que estos suponen. En este sentido, cabe preguntarse entonces si la nueva configuración del sistema y los desafíos que estos suponen también exige llevar a cabo modificaciones y reformas profundas al multilateralismo, tal como lo conocemos hoy en día.

\section{CRISIS PERMANENTE O CRISIS PARTICULAR}

Después de la Segunda Guerra Mundial, la Carta de Naciones Unidas fue negociada y ratificada en la ciudad de San Francisco y se estableció el Consejo de Seguridad como el organismo encargado de mantener la paz y seguridad internacional. Casi simultáneamente, en Bretton Woods se crearon el Fondo Monetario Internacional (FMI) y el Banco Mundial (BM) como instituciones para favorecer el desarrollo y la cooperación económica y posteriormente se conformó el GATT y su sucesor la OMC. Esto constituía un nuevo impulso para el modelo de instituciones internacionales que el Presidente Roosevelt había propuesto un par de décadas atrás. El fin de la guerra supuso un momento clave para el establecimiento de nuevas formas de cooperación interestatal entre los miembros de la comunidad internacional.

Ahora es importante señalar que este surgimiento y posterior proliferación de instituciones y regímenes multilaterales a nivel internacional ha promovido la convergencia de valores "mundiales" de una manera probablemente nunca antes vista en la historia. La mayoría de gobiernos en el mundo son democráticos, los derechos humanos son un concepto que, aunque todavía debatido en su significado integral, se defiende por gran parte de los Estados y durante el último siglo se ha visto el mayor crecimiento económico, incluyendo la reducción de la pobreza (Zedillo, 2003). Sin embargo, parece ser que en la práctica, el sistema multilateral no responde precisamente a dichos valores y, por el contrario, se evidencia unainsistencia de los Estados para actuar de manera unilateral en la defensa de sus intereses de seguridad y bienestar. En relaciones internacionales este dilema se ha planteado como el debate entre ganancias absolutas versus ganancias relativas. Algunos teóricos plantean que los Estados buscan maximizar sus ganancias totales (absolutas) sin importar cómo afecta su posición vis-a-vis la posición de los otros estados. En este sentido, mientras que las ganancias relativas tienen una característica comparativa en la que importa menos cuál es la ganancia real y absoluta sino cómo dichas ganancias han afectado la posición de cada Estado en relación a los otros miembros del sistema internacional (Mersheimer, 1995).

El propósito altruista del multilateralismo es establecer reglas de comportamiento internacional de mutua satisfacción para los países, así como el desarrollo de instituciones que favorezcan la cooperación internacional. La paz, más en la línea de lo que establecería Kant, requiere un acuerdo entre los Estados que establezca reglas claras y roles en el sistema internacional. Según autores como Keohane, las instituciones internacionales aumentan los niveles de transparencia entre los Estados y reducen los costos de transacción. Estos se constituyen en mecanismos de fomento de la confianza e inducen a la cooperación (Keohane, 1984). 
Lamentablemente, el estado que hemos vivido en las últimas décadas no es un verdadero multilateralismo, sino un unilateralismo disfrazado de multiplural.Varios países considerados potencias han hecho gravitar los asuntos de interés internacional en base a sus intereses nacionales particulares y mediante el proceso de coaliciones; las negociaciones internacionales han reflejado un sesgo hacia varios grupos de países sin una plena participación o legitimidad de todos los miembros de la comunidad internacional. "Una nación, un voto" no ha sido verdaderamente un principio que guíe a las organizaciones internacionales en los temas trascendentales de seguridad y bienestar.

Adicionalmente, el multilateralismo tiene serias deficiencias que se han agravado últimamente. La presencia de nuevas amenazas a la seguridad y la paz, así como la fuerte crisis que enfrenta el modelo económico que supuestamente habría generado la prosperidad, generan serios cuestionamientos a la capacidad de la estructura de gobernanza internacional para solventar o des-escalar conflictos. Las decepciones más recientes del sistema multilateral se ejemplifican en una marginalización o el desplazamiento hacia un rol secundario de las Naciones Unidas en temas de seguridad, las divisiones transatlánticas en los conflictos de Iraq y Afganistán (y la consecuente división en la OTAN), así como las divisiones en la misma Unión Europea y las trabas vistas en el proceso de Doha de la Organización Mundial de Comercio.

Por estas razones, el multilateralismo aparenta estar en una crisis. Ni la ONU ni los aliados ni la comunidad internacional impidieron la intervención de Estados Unidos en Iraq; la OMC no avanza en su ronda de Doha y la fricción entre grupos de países no logra resolverse, además de que muchas disputas comerciales se han politizado (Reich, 2005). Organismos como el FMl y el Banco Mundial pierden cada vez más su legitimidad en países del sur - gran parte de los países de la región han saldado sus deudas con ambos organismos.

Las críticas al sistema multilateral son extendidas. Aunque se puedan reconocer sus beneficios en reducir costos de transacción para los países y su capacidad para proveer bienes públicos internacionales, también se resalta su característica poco democrática y elitista. Su naturaleza burocrática (en el caso de instituciones), su separación de las instituciones democráticas nacionales y la limitada participación de ciudadanos o individuos debilitan su capacidad de rendir cuentas y de generar verdaderas deliberaciones en un sentido democrático (Dahl, 1999; Dahl Tufte, 1973; Rubenfeld, 2004).

De igual manera, las instituciones internacionales generan una dinámica elitista en donde pocos actores son capaces de influir en el proceso, a diferencia de los procesos democráticos en los que el desarrollo de consensos y la participación inclusive de grupos de interés o de cabildeo da forma a la política pública, construyendo formatos de diálogo más extendidos. La inclusión de ONGs y organizaciones de la sociedad civil, como la Cruz Roja, como observadores en Naciones Unidas, ha reducido este déficit en alguna medida, pero es evidente que la participación 
individual es extremadamente limitada y solo ciertas organizaciones internacionales llamadas de la sociedad civil son admitidas.

A pesar de lo dicho, los países, particularmente del sur, reconocen que sin cooperación recíproca de las naciones su capacidad individual para conseguir sus propias políticas domésticas en temas como seguridad, control de flujos ilícitos, mitigación de conflictos, manejo de la economía internacional y el control del calentamiento globales casi imposible de lograr (Keohane, Macedo, Moravscik, 2009).

En relación a temas de paz, conflicto y seguridad internacional, la multiplicación de organismos dedicados al mantenimiento de la paz, particularmente a nivel regional, así como el surgimiento de nuevos actores ${ }^{1}$, induce a nuevas oportunidades no vistas con anterioridad; sin embargo, también representan un gran riesgo para la intervención legítima y necesaria. Por su lado, la creación de instituciones o regímenes regionales permite la especialización, la asignación de recursos y la creación de soluciones locales sin injerencias geopolíticas externas, pero también existe el grave riesgo de duplicación de esfuerzos, la compleja coordinación de acciones conjuntas y hasta una posible competencia por protagonismo, especialmente porque las instituciones y sus autoridades tratan de mantenerlas vigentes o relevantes (Koops, 2010).

La debilidad del sistema multilateral internacional para responder a los serios problemas que tienen repercusiones sobre la comunidad internacional, el emergente rol de nuevos actores estatales y no gubernamentales con una clara intencionalidad de incidir y hasta reformar la estructura de la política exterior mundial, la creación de nuevos bloques y el fortalecimiento de otros bloques regionalesreflejan una transformación de las condiciones mundiales frente a la falta de beneficios provisto por la estructura multilateral actual, y nos permite considerar que existe una crisis en el sistema.

La limitada respuesta de las instituciones que conforman Naciones Unidas a la mayoría de temas antes planteados ha llevado a la circunvalación de estas instituciones, prefiriendo significativamente mecanismos ad hoc como el G-8 o el G-20; sus miembros argumentan que estos mecanismos hace que sea más efectiva la toma de decisiones; sin embargo, se reduce su legitimidad democrática al ser excluyente. El propio sistema de Naciones Unidas tiene una manifestación interna ejemplificadora de la crisis que sufre frente la imposibilidad de reformar el Consejo de Seguridad y de revitalizar la Asamblea General con el propósito de otorgar una mayor y más equitativa participación de todos los actores internacionales de manera acorde con la nueva realidad y ya no la que imperaba en $1945^{2}$.

1. Grupos insurgentes y/o terroristas, así como organizaciones de la sociedad civil, ONGs y transnacionales.

2. El autor fue panelista en el Taller "Crisis del Multilateralismo" organizado por la Facultad Latino Americana de Ciencias Sociales y la Fundación Friedrich Ebert, mayo, 2012 en FLACSO sede Quito; las ideas vertidas allí han nutrido este artículo. 


\section{CAMBIOS DRÁSTICOS EN EL SISTEMA INTERNACIONAL}

El escenario estratégico global ha variado significativamente. La unipolaridad estadounidense del sistema se muestra cuestionada por potencias emergentes en temas económicos pero también en el ámbito militar. China en particular, pero también los otros miembros delgrupo llamado BRIC (Brasil, Rusia, India, China), han aparecido rápidamente en el sistema y han impuesto su marca; inclusive actores no tradicionales como las organizaciones no gubernamentales adquieren cada vez mayor influencia y hay nuevos retos que enfrenta el sistema de una manera colectiva que han surgido en los últimos años, como la seguridad alimentaria, cambios en la matriz energética mundial, el calentamiento global - asuntos que requieren de una acción colectiva y mayoritaria para solucionarlos de una forma responsable. Ante esta situación ha sido evidente la dificultad de las organizaciones multilaterales globales tradicionales, la ONU y su sistema, para definir e implementar soluciones.

La última década ha visto cambios importantes en las características y dinámicas estratégicas mundiales y regionales; la distribución de los recursos naturales en las diferentes regiones, los cambios en las relaciones bilaterales estratégicas, la reconfiguración en torno a qué países constituyen las mayores economías y la creciente proliferación de nuevos foros multilaterales del sur global son algunas de estas nuevas características. El sistema internacional actual diverge significativamente en temas económicos, geopolíticos y organizativos, así como en los actores que lo conforman en comparación con el sistema que teníamos en la pos caída del muro de Berlín. Estos cambios tienen consecuencias importantes en la capacidad de los organismos internacionales para proveer bienes públicos aceptados y legítimos ante la comunidad internacional. A continuación se describen algunos de los cambios trascendentes que tienen impacto sobre la gestión multilateral en temas de seguridad internacional ${ }^{3}$.

Por un lado, el crecimiento económico de los países antes llamados de la periferia es un cambio fundamental en las relaciones geo-económicas y geo-políticas, lo que pone a países como China, Brasil, India y Rusia entre las economías más grandes y de mayor crecimiento; en 2012 estos países representaron un 25\% de la producción mundial (The Economist, 2013). El crecimiento de mercados dinámicos y grandes en Asia - particularmente China y la India - pero también los demás miembros de los BRICS ha resaltado la prioridad de los temas relacionados con recursos en la agenda de seguridad internacional ${ }^{4}$. Altos niveles en los precios y la demanda de commodities ha llevado a los gobiernos, las industrias, la sociedad civil y a los mismos organismos internacionales a lanzar iniciativas para contrarrestar este vínculo negativo entre los temas relacionados a

3. Aunque los elementos analizados en este artículo se consideran fundamentales, de ninguna manera son un análisis exhaustivo y completo.

4. Solo para nombrar un ejemplo, lo sucedido en Sudán resalta la difícil mezcla entre política, seguridad y recursos naturales. 
recursos y la aparición de conflictos; se incluye además el desarrollo de sistemas tempranos de alerta y de monitoreo de conflictos como elementos para mitigar los efectos adversos y sobre todo para incluir el manejo correcto de recursos en los procesos de construcción de paz (SIPRI, 2011).

Algunos conceptos entran al sistema internacional como la responsabilidad de proteger (R2P) y mediante ésta se justifica la intervención en algunos países. R2P se basa en el principio de que la soberanía implica responsabilidades para los Estados, particularmente la de proteger a los ciudadanos, y en caso de que el Estado no pueda o no esté dispuesto a proteger a sus ciudadanos por causas de guerra, insurgencia o incapacidad, es la responsabilidad de la comunidad internacional precautelar por aquellos que sufren (Oficina del Consejero Especial para la Prevención del Genocidio, 2001). La falta de acción ante los graves casos de violación de derechos humanos en Somalia en 1993, Ruanda en 1994 y Bosnia en 1995 denotó que la comunidad internacional abdicaba su responsabilidad compartida y fue un simple espectador de las atrocidades (Evasn \& Schanoun, 2002). Los promotores de este concepto argumentan que su fundamento se basa en la soberanía de los Estados, el artículo 24 de la Carta de Naciones Unidas (responsabilidades del Consejo de Seguridad), la declaración de derechos humanos, las leyes humanitarias, así como las práctica de los Estados, los organismos regionales y el Consejo de Seguridad (derecho consuetudinario) (Oficina del Consejero Especial para la Prevención del Genocidio, 2001). Sin embargo, la intervención humanitaria tiene muchos detractores, inclusive las organizaciones no gubernamentales dedicadas a las causas humanitarias, principalmente por la mezcla de la acción militar en temas humanitarios.

La responsabilidad de proteger también tiene su arista positiva ya que cambia la dinámica de análisis de los actores involucrados de los gobiernos respecto a los ciudadanos y las ciudadanas y sus necesidades; asegura que el actor principal en la obligación de proteger a sus ciudadanos sea el Estado mismo y finalmente R2P incluye no solo la responsabilidad de reaccionar, sino de prevenir el escalamiento, así como la responsabilidad de reconstruir. La respuesta de la comunidad internacional ante la crisis en Haití luego del terremoto ejemplifica este último conceptode una manera amplia. El problema no está en el principio de R2P, sino en la aplicación del mismo y en muchos casos en la falta de legitimidad para su aplicación.

Por su parte, el principio de autodeterminación sigue evolucionando y se convierte en la lucha de grupos sub-nacionales para obtener autonomía completa. Originalmente esbozado en los catorce principios del Presidente Wilson, la autodeterminación se concebía como aplicable a los países europeos y no a las colonias; esta situación se dio hasta la Declaración de Independencia de los Pueblos Coloniales de Naciones Unidas en 1960, en la que la auto-determinación pasa de ser un principio a un derecho de los pueblos (Brahm, 2005). Mediante este concepto, muchas naciones vieron nacer su independencia del yugo colonial. El derecho a la autodeterminación, en relación a la jurisdicción territorial se convirtió prominente con el aval de la Corte Internacional de Justicia y la jurisprudencia que ésta creó (Corte Internacional de Justicia, 
1971, 1986, 1995) ${ }^{5}$. Sin embargo, como se reflejó en Europa Central y del Este y todavía sucede en algunos enclaves en el África, la autodeterminación se da principalmente por elementos nacionalistas, que lleva a muchos grupos étnicos a buscar sus propias naciones (Brahm, 2005). Esto transforma al concepto de autodeterminación que se esbozó en la Carta de Naciones Unidas de un proceso de descolonización a un proceso que fomentó conflictos inter e intra-estatales (o al menos les entregó legalidad), con serias implicaciones para el orden mundial.

En ese sentido, las guerras pasan de ser entre Estados a ser (primordialmente) intra-estatales y también existe una evolución en cómo se dan estas guerras civiles. Más de la mitad de ellas resurgen luego de pasar 5 años de acuerdos (Wallensteen \& Sollenberg, 2001). Esto también está relacionado, como varios estudios identifican, con los procesos de democratización que han experimentado estas nuevas naciones, en donde las instituciones todavía son débiles y los mecanismos de pesos y contrapesos instaurados no son lo suficientemente fuertes para prevenir el uso de violencia y zanjar las discrepancias entre los grupos sociales (Chege, 1995; Young, 1999). En los Estados débiles, el Estado es gobernado más por poder e imposición que por consenso y legitimidad de los ciudadanos, además de que la autoridad central es enfrentada por grupos internos (Olson \& Soederberg, 2002).

Otro desarrollo es el impulso cada vez mayor para reformar el Consejo de Seguridad de la Organización de Naciones Unidas. Varios países, incluyendo Japón, Alemania, Brasil e India, son los mayores contendores para convertirse en miembros permanentes del Consejo. De estos candidatos, India ha recibido el aval de Estados Unidos para conseguirlo. Se han esbozado modelos para reformar el Consejo que buscan desde una mayor flexibilidad en la rotación de los miembros hasta una total eliminación del veto de los cinco miembros permanentes. Lo que queda claro es que aunque hay un impulso fuerte para su reforma, no es tan clara una reforma profunda al Consejo, inclusive cuando su estructura de poder y de toma de decisiones es quizás el mayor reflejo de la dinámica geo-política de la posguerra que no refleja la situación del nuevo siglo.

Por su parte, las redes sociales se han desarrollado como un mecanismo con una capacidad tremenda para movilizar a las masas, pero también han permitido hacer más internacionales a temas antes considerados no relevantes. Esto ha democratizado en alguna medida el acceso al diálogo y la participación de ciudadanos que tradicionalmente se veían excluidos de los procesos políticos. Sin lugar a duda, un claro ejemplo de la capacidad de convocatoria y movilización mediante esos mecanismos es la Primavera Árabe, en la que el mundo tuvo acceso no sólo a medios de comunicación privados y públicos, sino a manifestaciones expresas de individuos en medio de la situación. Este es el nuevo efecto CNN y es un desarrollo muy positivo.

5. La jurisprudencia sentada incluye: Caso concerniente a Burkina Faso y Mali,Disputas de Fronteras (1986). Reportes Corte Internacional de Justicia; Legal Consequences for States of the Continued Presence of South Africa in Namibia (South West Africa), (1971), sin importar la resolución del Consejo de Seguridad 276; Caso concerniente a Timor del Este (Portugal v. Australia), (1995). 
Sin lugar a duda, otro cambio importante es la inestabilidad y la crisis de la economía internacional. La crisis financiera se da en el "centro" y son realmente los países de la "periferia" quienes sostienen al centro. Brasil, India, China y Rusia han comprometido sendos recursos para potenciar la capacidad del Fondo Monetario Internacional (FMI) en la cumbre del G-20 en México en junio de 2012, algo que no sólo establece la capacidad económica y solvencia de estos países emergentes, sino la interdependencia y el reconocimiento de que los efectos negativos de una profundización de la crisis serían perjudiciales para estos países y su sociedades. China comprometió 60,000 millones de dólares, mientras que los otros tres países 10,000 millones de dólares cada uno (Reuter, 2012a). Lamentablemente, esto también significa que importantes recursos que se podrían destinar para el mayor desarrollo y para atender las profundas necesidades de políticas sociales en estos países son destinadas para financiar aquellos sectores económicos y aquellos países que ocasionaron esta crisis.

Asimismo, la crisis económica ha significado un replanteamiento de los gastos en materia de seguridad y defensa, principalmente en Europa. El Reino Unido reducirá aproximadamente un veinte por ciento de su ejército en los siguientes ocho años como parte de una reforma estructural que según el Ministro de Defensa británico llevará a unas fuerzas más "equilibrada, capaz y adaptable"(El Universo, 2012). Otros países como Italia también proyectan reducciones significativas, de alrededor del 10 por ciento (El Pais, 2012). En general, la Unión Europea aspira a una reorientación de sus fuerzas armadas nacionales para una mayor especialización, de acuerdo a las ventajas competitivas e institucionales de cada país miembro. Sin embargo, con la reorientación hacia el Asia-Pacífico de Estados Unidos y el rol de facto de brazo ejecutor de mandatos del Consejo de Seguridadque ha asumido la OTAN, queda la duda sobre su verdadera orientación y su misión a nivel estratégico. Igualmente, se evidencia una merma en la solidaridad de la seguridad colectiva después del agotamiento de la intervención en Afganistán y la división ante la intervención en Libia, en donde pocos fueron los países que participaron y Estados Unidos prefirió "Iiderar desde atrás". Como el Grupo de Estudios Estratégicos de España menciona, "las divergencias estratégicas se acentúan y los ajustes y la austeridad ante la crisis económica ahonda en la supeditación de lo colectivo a lo puramente nacional" (GEES, 2012:2).

Asimismo, Estados Unidosha reorientado sus fuerzas hacia el Asia Pacifico y ha insinuado que Europa debe solventar su necesidad de seguridad (Reuters, 2012b). La reorientación de las fuerzas militares hacia el AsiaPacífico se da al mismo tiempo que se produce un recorte en el presupuesto militar estadounidense y mientras se negocia la Alianza Trans-Pacífico ${ }^{6}$, lo que significa un cambio importante en la orientación geo-estratégica de ese país y no una expansión de

6. La Alianza Trans-Pacífico es un acuerdo de libre comercio que se encuentra en proceso de negociación e incluye a Nueva Zelanda, Chile, Singapore y Brunei como miembros fundadores y a quienes Estados Unidos, Perú, Australia y Vietnam se han unido. México y Canadá recientemente expresaron su interés por ser incluidos en el proceso. Este acuerdo comercial es una muestra más de la reorientación estratégica hacia el Asia-Pacífico de Estados Unidos. 
su esfera de influencia. Como es de esperarse, autoridades del gobierno chino han expresado su preocupación. El vicepresidente chino Xi Jinpin expresó en su última visita a Estados Unidos que "en tiempos cuando la gente aspira a la paz, estabilidad y desarrollo, darle prominencia deliberadamente a la agenda de seguridad militar, escalar la movilización militar y fortalecer las alianzas estratégicas no es lo que la mayoría de países en la región esperan"(Dyer, 2012). Esta reorientación muy probablemente tenga consecuencias y efectos que se verán en las siguientes décadas y que podrían llevar a escalar las tensiones entre China y Estados Unidos.

\section{DESAFÍOS DEL SISTEMA}

Uno de los principales desafíos que enfrenta el multilateralismo es la gran divergencia de intereses y posiciones del sistema internacional. Definir términos de seguridad y defensa dentro de las organizaciones dedicadas al tema ha demostrado ser una tarea complicada. El terrorismo suele ser el clásico ejemplo, pero en general la definición de amenazas y factores de riesgo enfrenta la misma dificultad. Inclusive entre organizaciones como la ONU, la OTAN, y la OSCE, que tienen liderazgos similares y que producen listados similares, su priorización de temas es diferente.

Otro desafío importante que tiene relación con lo anterior es que la contraposición tan fuerte de intereses ha hecho que el éxito de estas organizaciones se mida por su capacidad para llegar a un acuerdo y no por el verdadero impacto que tengan estas decisiones o su capacidad cierta para resolver los problemas de seguridad internacional. El caso de la proliferación nuclear es particularmente un buen ejemplo, pero temas como la independencia de Sudan del Sur también nos muestran las dinámicas que enfrentan estos organismos.

Como Crocker (et al,2011) han manifestado, aunque las potencias emergentes tienen intereses globales que manifiestan claramente y existen evidencias de cómo sus preferencias y posiciones han cambiado el discurso e inclusive las prioridades internacionales, sin embargo, no están quizás preparadas para enfrentar directamente las responsabilidades de liderazgo y de financiamiento que representaría asumir el orden internacional, particularmente relacionado con la seguridad internacional. Esta dinámica se da a la par que las instituciones internacionales sufren de la dicotomía entre legitimidad y eficiencia. Esto deja entrever posiblemente mayores obstáculos para la gobernanza mundial si se crea un vacío de liderazgo. Por ahora se han visto indicios de voluntad de los países emergentes en temas económicos como se mencionó anteriormente, pero en temas de seguridad internacional esto todavía no se ha esclarecido.

Otro desafío que enfrenta el multilateralismo son las relaciones bilaterales y sobre todo la regionalización del sistema. Las relaciones bilaterales son másrápidas y alcanzar el consenso entre dos partes es mucho más rápido; sin embargo, no siempre resulta en la solución más parsimoniosa. La falta de percepción de una amenaza estructural tras el final de la Guerra Fría lleva a una fragmentación de la seguridad europea. El resultado es la deriva estratégica de la 
OTAN y el estancamiento de los mecanismos para desarrollar la política de defensa europea que introdujo el Tratado de Lisboa frente a acuerdos bilaterales como los firmados recientemente entre Francia y Reino Unido.

Por su parte, la regionalización del mundo responde en parte a una reacción al obstruccionismo existente en aquellas instituciones que deberían ser el foro mundial de los asuntos o el centro del multilateralismo. Estos foros regionales en sí no son necesariamente negativos o contrapuestos al multilateralismo, pero sí generan dinámicas cerradas en donde se crean normas, reglas y formas de toma de decisión a su interior,aunque bien estas pueden ser contraproducentes porque no siempre son compatibles con aquellas que se acostumbran en foros globales. Por otro lado, la regionalización tiene sus propios beneficios en la medida que disminuye el ruido en las conferencias internacionales al presentar a estos bloques más cohesionados.

\section{ELEMENTOS NECESARIOS PARA FORTALECER EL MULTILATERALISMO}

En definitiva, para que el multilateralismo funcione deben existir algunos cambios profundosen las instituciones como el FMI o el Consejo de Seguridad de Naciones Unidas, pero los objetivos como la paz y la estabilidad exigen algo más que cambios cosméticos para dar cabida a potencias emergentes. El mundo del siglo XXI es demasiado interdependiente y complejo para ser reorganizado exclusivamente en claves de soberanía. Un multilateralismo moderno exige un nuevo marco de cooperación que, además de los inevitables equilibrios de poder, tenga en cuenta la diversidad de los desafíos actuales y la necesidad de reafirmar un modelo normativo sobre una base democrática y de respeto a los derechos humanos.

Definitivamente el punto central para fortalecer el multilateralismo es generar una mayor legitimidad de los procesos de toma de decisión, sean estos por procedimientos adhoc (regímenes) o principalmente por aquellas instituciones llamadas a salvaguardar la paz y seguridad internacionales. Esta legitimidad es esencial para cubrir el déficit democrático antes mencionado, pero también para reducir la divergencia entre eficacia y legalidad.

De igual manera, se deben generar mecanismos de diálogo y consulta, así como otras herramientas muy comunes en la disciplina de resolución de conflictos para integrar a más actores que no sean sólo los Estados. El objetivo debe ser la verdadera democratización de los foros multilaterales para permitir que las opiniones y necesidades de los ciudadanos sean manifestadas e incluidas. El proceso de Ottawa contra el uso, producción y manufactura de las minas antipersonales demostró que las organizaciones de la sociedad civil pueden generar procesos -inclusive paralelos a Naciones Unidas- y provocar cambios radicales a prácticas nefastas pero muy arraigadas. No obstante, se debe propender a incluir no sólo a las ONGs internacionales que son cada vez actorescon mayor reconocimiento, sino también a los pueblos de las naciones en una forma más flexible y heterogénea. 
Por su parte, la fuerte crítica a la intervención de Estados Unidos en Iraq, así como el retiro progresivo de varios países de las coaliciones,son muestras de que el unilateralismo ha perdido terreno y afortunadamente conceptos como la guerra preventiva -inclusive al interior de Estados Unidos - no han calado (del todo). Sin embargo, las acciones unilaterales seguirán siendo la sombra que persiga al multilateralismo, particularmente en temas considerados de alta política internacional o high politics.

Finalmente, cualquier reforma a las instituciones y regímenes internacionales debe ser más inclusiva con las potencias emergentes y su nuevo rol en el mantenimiento de la paz. No se puede demandar una mayor participación sin permitir que estas potencias emergentes tengan mayor capacidad de influir sobre los procesos y la toma de decisiones. Sin embargo, no se puede dejar de lado que las asimetrías, especialmente en capacidad bélica y de disuasión, siguen siendo radicales y aunque el sistema deba cambiar, las asimetrías deben ser incluidas en los nuevos diseños para asegurar que los actores claves tengan interés en permanecer y fortalecer esas instituciones.

Debido a la centralidad de las potencias emergentes, como actores relevantes del sistema internacional, pero también como artífices de los cambios estructurales del mismo, es necesario que se establezcan los nuevos lineamientos de lo que se establece en esta transición a un sistema crecientemente multipolar. La experiencia de los últimos años parecería indicar que esta transición incluiría una alta dosis de continuidad - por el momento no hay un cambio radical sino una situación de inclusión y reforma de las instituciones existentes. Contrario a formulas anteriores, como el Concierto de Viena o la creación del Consejo de Seguridad con sus 5 miembros permanentes, la transición de poder no ha significado un desbalance profundo de la paz mundial ni se ha sucedido por una inestabilidad en el sistema de seguridad mundial. Sin embargo, esta reestructuración de los centros de poder mundiales a partir de la implosión soviética requiere que las potencias emergentes no demanden simplemente una posición de mayor liderazgo, sino que deben estar dispuestas a incurrir en los costos políticos y económicos que significa desarrollar esta nueva infraestructura de gobernanza a nivel regional y a nivel sistémico.

Las reformas a las estructuras de gobernanza que reconozcan principalmente el nuevo rol y condición de poder que estas potencias emergentes representan permitirían sostener un mejor manejo de una paz (negativa) del sistema internacional. La alternativa podría significar una necesidad de las potencias emergentes de redefinir el sistema, poniendo en juego la tan inestable "estabilidad" del sistema actual.

Las potencias emergentes deben asumir un rol de liderazgo y afrontar los costos implícitos con el nuevo rol que les compete. En alguna medida la proliferación de instituciones regionales refleja sus diseños de política exterior, sus aspiraciones regionales/internacionales y sus acciones geoestratégicas; sin embargo, para poder posicionarse como verdaderos miembros del sistema deben enfocar sus acciones en foros multilaterales internacionales.Si éstas mantienen una política exterior para establecerse sólo dentro de foros regionales (UNASUR, el Grupo de Shanghái, 
etc.), dejando de lado organismos multilaterales más plurales (e internacionales) su capacidad de sostenerse como actores relevantes del sistema es definitivamente limitada.

En definitiva, el sistema multilateral se parece a la democracia, es el menos malo de los sistemas de gobernanza internacional.

\section{REFERENCIAS}

- $\quad$ Reuters (2012a, 18 de junio). BRICS acuerdan fortalecer el FMI (18 de junio 2012). Recuperado el 30/06/2012 de http://www.aztecanoticias.com.mx/notas/finanzas/118097/bricsacuerdan-fortalecer-al-fmi

- $\quad$ El País (2012, 6 de julio). El Gobierno italiano aprueba un decreto para ahorrar 26.000 millones en tres años. Recuperado el 6/07/2012 de http://internacional.elpais.com/internacional/2012/07/06/actualidad/1341539784_751680.html

- $\quad$ El Universo (2012, 2 de julio). El Reino Unido anuncia la mayor reforma de sus fuerzas armadas en décadas. Recuperado el 5/07/2012 de http://www.eluniverso.com/2012/07/ 05/1/1361/reino-unido-anuncia-reforma-sus-fuerzas-armadas-decadas.html

- $\quad$ Reuters (2012b, enero). New Pentagon strategy stresses Asia, cyber, drones. Recuperado el 26/06/2012 de http://www.reuters.com/article/2012/01/05/us-usa-military-obamaidUSTRE8031Z020120105

- $\quad$ The Economist (2013, 6 de mayo). Welcome to the post-BRIC world.

- Grupo de Estudios Estratégicos (GEES), (2012, julio). Alerta Estratégica. Recuperado el 10/ 07/2012 de www.gees.org

- Brahm, E. (2005).Self Determination Procedures. Recuperado el 20/07/2012 de http:// www.beyondintractability.org/bi-essay/self-determination

- $\quad$ Chege, M.(1995). Between Africa's Extremes.Journal of Democracy, 6 (1:44-51)

- $\quad$ Chester, C., Osler, F., Hall, P. (2011).Collective Conflict Management: a new formula for global peace and security cooperation.International Affairs, 87 (1:39-59)

- $\quad$ Crawford, Y (1999). The Third Wave of Democratization in Africa: Ambiguities and Contradictions. En: R. Joseph(ed.),State, Conflict, and Democracy in Africa, Boulder: Lynne Rienner Publishers. 
- $\quad$ Dahl, R.(1999). Can International Organizations be Democratic? A Skeptic's View.En: I. Shapiro and C. Hacker-Cordón (eds.), Democracy's Edges, Cambridge: Cambridge University Press.

- $\quad$ Dahl, R. and Tufte, E. (1973).Size and Democracy. California: Stanford University Press.

- Dyer, G. (2012, 14 de febrero). US defends Asian Military Strategy. Financial Times. Recuperado el 29/06/2012 dehttp://www.ft.com/intl/cms/s/0/6ba83b3e-5738-11e1869b-00144feabdc0.html\#axzz1zCn0ub78

- $\quad$ Evans, G. et all, (2001), The Responsibility to Protect. International Commission on Intervention and State Sovereignty Recuperado el 2/07/2012 de http://responsibilityto protect.org/ICISS\%20Report.pdf

- $\quad$ Frontier Disputes(1971, 1986, 1995).Reportes de la Corte Internacional de Justicia; Recuperado el 25/07/2012 de http://www.timdavis.com.au/Papers/Acqusition_ of_Territorial_Sovereignty.pdf].

- $\quad$ Gareth, E. andShanoun, M. (2012). The Responsibility to Protect. Foreign Affairs, 81 (6) 99-110.

- $\quad$ Gilpin, R.(1981).War and Change in World Politic. New York: Cambridge University Press.

- $\quad$ Kardas, S.(2001). Humanitarian Intervention: The evolution of the idea and practice. Journal of International Affairs, 4 (2) 27-37.

- $\quad$ Keohane, R.(1990). Multilateralism: An Agenda for Research.International Journal, 45 731-764.

- Keohane, R. (1984). After Hegemony: Cooperation and Discord in the World Political Economy. Princeton, NJ: Princeton University Press.

- $\quad$ Keohane, R., Macedo, S. y Moravcsik, A. (2009). Democracy Enhancing Multilateralism. International Organization, 62 1-31

- $\quad$ Koops, J. (2010). Effective Miltilateralism in Peacekeeping, Capacity-Building, and Crisis Management: The Challenge of Inter-organizational Cooperation. Recuperado el 20/06/ 2012 de [http://www.effectivemultilateralism.info/library/Focus2010-1_Koops.pdf]

- Mersheimer, J. (1994-1995). The False Promise of International Institutions. International Security, 19 (3) 5-49 
- Naciones Unidas (2001), The responsibility to Protect, Oficina del Consejero Especial para la Prevención del Genocidio Recuperado el 02/07/2012 de [http://www.un.org/en/ preventgenocide/adviser/responsibility.shtml

- Olson, T., y Soederberg, M. (2002).From Intra State War to Democratic Peace in Weak States.Suecia:Universidadde Uppsala.Recuperado el 30/07/2012 de http://www.pcr.uu.se/ digitalAssets/18/18593_UPRP_No_5.pdf

- $\quad$ Reich, A. (2005).The Threat of Politicization of the World Trade Organization.Bar Ilan Univ. Pub Law Working Paper, No. 7-05

- $\quad$ Rubenfeld, J.(2004). Unilateralism and Constitutionalism.New York University Law Review,79 (6) 1971-2028.

- Ruggie, J.(1992).Multilateralism: the anatomy of an institution.International Organization, 46.(3), 561-598.

- $\quad$ SIPRI, (2011). Yearbook.Recuperado de http://www.sipri.org/yearbook/2011/02

- Wallensteen, P. and Sollenberg, M. (2001). Armed Conflicts, 1989-2000.Journal of Peace Research, 38 (5)629-644.

- Zedillo, E.(2003). Roosevelt was right about Multilateralism.Yale Global Online. Recuperado el27/06/2012 de http://yaleglobal.yale.edu/content/roosevelt-was-right-aboutmultilateralism 